Results Among 4,484 PLWH (1632 whites, 2676 blacks, 176 other races; 1031 women, 3429 men, 24 transgenders) over a total follow-up of 22188 years, there were 43 ACs (all men, 24 whites, and 18 blacks). The median age at AC diagnoses was $52 ; 36$ were men who have sex with men (MSM) and 7 were heterosexual men $(\mathrm{P}<0.0001)$. The incidence was 267.2 cases per 100,000 men each year. Being male $(\mathrm{P}<0.0001)$, MSM $(P<0.0001)$, white $(p=0.03)$, aged $45-54$ years $(p=0.02)$, and with low nadir CD4 $(p<0.0001)$ were independently associated with AC.

Conclusion AC incidence among HIV-infected men was 148.4 times higher with earlier onset than general populations. However, public awareness of AC screening, specifically among the targeted population is lacking. This study can be valuable for the development of related screening programs and clinical practice guidelines for PLWH in the region.

Disclosure No significant relationships.

\section{P189 EVALUATION OF APTIMA HIV-1 QUANT ASSAY PERFORMANCE USING PLASMA AND DRIED BLOOD SPOTS}

Julie Nelson*_Kara Compliment, Paul Alabanza, Dana Lapple, Takesha Mcmillion, Marcia Hobbs. University of North Carolina at Chapel Hill, Microbiology and Immunology, Chapel Hill, USA

\subsection{6/sextrans-2019-sti.341}

Background The Hologic Aptima HIV-1 Quant Assay that is currently available on the Panther system is FDA-cleared for plasma viral load monitoring. We compared to other assays for use with dried blood spots (DBS) and for early infant diagnosis (EID) using plasma and DBS.

Methods Spiked dilution panels of plasma and DBS were made using negative donor blood and subtype B and subtype C HIV-1 stocks. Patient samples included plasma from HIV-1infected adults, plasma from HIV-1-infected infants and exposed uninfected infants, and DBS from infected adults. Samples were run on Aptima Quant and either Abbott RealTime HIV-1 Quant assay or Hologic Aptima HIV-1 Qual assay.

Results Aptima Quant and Abbott Quant were comparable: the $95 \%$ limit of detection was $42 \mathrm{cp} / \mathrm{ml}$ for Aptima Quant and $91 \mathrm{cp} / \mathrm{ml}$ for Abbott Quant. EID comparisons showed that Aptima Quant and Qual had 100\% sensitivity and 98.9\% specificity with overall agreement between the assays of $98.4 \%$. Aptima Qual had a slightly lower limit of detection $(95 \%$ limit of detection was $27 \mathrm{cp} / \mathrm{ml}$ for Aptima Qual and $65 \mathrm{cp} / \mathrm{ml}$ for Aptima Quant). With spiked DBS, all 16 DBS with $>30 \mathrm{cp} / \mathrm{ml}$ on Aptima Quant were detected on Aptima Qual, 10/12 (83\%) with '<30 detected' on Aptima Quant were detected on Aptima Qual, and 2 of 14 (14.3\%) with 'not detected' on Aptima Quant were detected on Aptima Qual. Among 200 DBS from infected adults, overall agreement between the Aptima Quant and Aptima Qual was 90\% when '<30 detected' was counted at positive. 13 DBS from uninfected adults were negative on both assays.

Conclusion The Hologic Aptima HIV-1 Quant assay performed similarly for viral load and EID on both plasma and DBS samples. Our data suggest the ' $<30$ detected' result could be used as the indeterminate range for Aptima Quant using DBS, as recommended by the new WHO guidelines.

Disclosure No significant relationships.

\section{P190 THE ROLE OF HELPLINE COUNSELING IN HIV STATUS DISCLOSURE AMONG SEXUAL PARTNERS: A CASE STUDY OF TOLL FREE HELPLINE IN UGANDA}

Julius Ssekinkuse*. Communication for Development Foundation Uganda, Counseling, P.O. Box 8734 Kampala, Uganda, Uganda

\subsection{6/sextrans-2019-sti.342}

Background Globally, 35.3 million people were living with HIV at the end of 2012. Sub-Saharan Africa remains most severely affected, with nearly 1 in every 20 adults living with HIV and accounting for $71 \%$ of the people living with HIV worldwide. Uganda has sustained some impressive response to HIV/AIDS epidemic grounded in a multi-sectoral approach coordinated by Uganda AIDS Commission. The response to stop and control HIV/AIDS has yielded many useful strategies and yet mistakes and missed opportunities. HIV/AIDS continues to be a major socio-economic challenge and is among the leading causes of morbidity and mortality given the increasing new infections due to status non-disclosure among sexual networks. Feedback from the Helpline indicate that those infected fear to disclose their HIV status to their sexual partners for many reasons thus the Helpline comes in to fill this gap through telephone counseling with timely responses Methods Data from Telephone calls received from those fearing to disclose their HIV status to their sexual partners was reviewed. A random selection of feedback responses was sampled to find out how the Helpline was helping the community deal to with the problem

Results Sampled 400 positive feed backs from community members who had benefited from Helpline counseling revealed that timely telephone counseling empowered them to disclose their HIV status to their sexual partners because of the prevention benefits discussed with Helpline counselors. The helpline empowered and educated callers about HIV and other STIs status disclosure benefits especially prevention of new infections

Conclusion The results of this study reveal that Helpline counseling can be a helpful resource in HIV/STIs prevention by empowering those infected to disclose their status with their sexual partners to protect and prevent new infections.

Disclosure No significant relationships.

\section{P191 SOUTH INDIAN LONG-DISTANCE TRUCK DRIVERS STILL AT HIGH-RISK FOR HIV/STI}

${ }^{1}$ Karl Krupp*, ${ }^{2}$ Kavitha Ravi, ${ }^{2}$ Anisa Khan, ${ }^{2}$ Vijaya Srinivas, ${ }^{2}$ Poornima Jayakrishna, ${ }^{2}$ Rishika Gupta, ${ }^{2}$ Neha Joshi, ${ }^{3}$ Purnima Madhivanan. ${ }^{1}$ Florida International Univeristy, Department of Health Promotion and Disease Prevention, Robert Stempel College of Public Health, Miami, USA; ${ }^{2}$ Public Health Research Institute of India, Prerana Women's Health Initiative, Mysore, Karnataka, India; ${ }^{3}$ Florida International University, Epidemiology, Miami, USA

\subsection{6/sextrans-2019-sti.343}

Background In a typical year, it is estimated that there are about 5 million long-distance truck drivers delivering goods on 3.3 million kilometers of roadways in India. Due to their high-risk behavior, truckers have long been considered a bridge population associated with the spread of sexually transmitted infection (STI) including HIV. The last national study of HIV prevalence in Indian truck drivers, completed in 2008, found an overall HIV prevalence of $4.6 \%$ and $2.7 \%$ for syphilis among long-distance truck drivers. 